\title{
III
}

\section{Theory and Modelling}


THE GALACTIC GRAVITATIONAL POTENTIAL

\author{
M. CREZE \\ Observatoire de Strasbourg, CNRS URA 1280 \\ 11 , rue de l'Université \\ 67000 Strasbourg, France
}

\begin{abstract}
Observational constraints on the galactic potential and modelling aspects are reviewed. The following conclusions come out. The mass distribution generating the galactic gravitational potential should include a bulge to account for the innermost features of the rotation curve, an extensive massive corona responsible for a flat rotation at large radius, and a disc able to produce a rotation curve as given by Rohlfs and Kreitschmann (1988) and a vertical force law $K_{z}$ as from Bienaymé et al. (1987). We still know very little about the potential more than $1500 \mathrm{pc}$ above the galactic plane.
\end{abstract}

\title{
1. WHAT ARE POTENTIALS GOOD FOR, HOW CAN WE GET THEM
}

This talk is not meant to address in great detail the complex mathematical problem of modelling the galactic potential. I would like to focus on more practical questions: How much do we actually know about the galactic potential? What are the most stringent observational constraints so far available? Within which limits is the galactic potential bound?

Gravitational potentials are of practical importance in two respects. First, they follow the trend of the total mass distribution, given by the Poisson equation

$$
\nabla^{2} \Phi=4 \pi G \rho .
$$

Second, they govern the motions of particles and gas on large scales. The relevant equation is then the Boltzmann equation, which we write here in its collisionless form

$$
v_{x} \cdot \frac{\partial f}{\partial x}+\frac{\partial \Phi}{\partial x} \cdot \frac{\partial f}{\partial v_{x}}+\ldots(y, z) \ldots=0
$$

where $f\left(v_{x}, x, \ldots\right)$ is the phase space distribution of the particles.

One possible approach would be to observe the mass distribution $\rho$, then to integrate (1) to get the force law $\nabla \Phi$ and then study the behaviour in this force field of any specific population, for instance the interstellar matter away from 
the galactic plane, which is the subject of this symposium. Unfortunately $\rho$ is usually not observable since some of the mass happens to be dark. So investigators would rather search for tracer populations which have a distribution function in the phase space $f$ that can be observed in situations simple enough to recover $\nabla \Phi$ through equation (2). Then equation (1) would provide an estimate of the total dynamical mass. One major difficulty in this process follows from equation (2). The Boltzmann equation sets a local dependence between the components of the force and the derivatives of the tracer distribution function with respect to space and velocity. This means that wherever the tracer sample is too small or too noisy to estimate the derivatives of the phase space distribution, we get no information about the force field at this place, neither do we get any information about the local density from (1).

The general trend of ideas initiated by J. Oort (1932) has been to collect pieces of evidence that might help choosing a realistic mathematical formula describing either $f$ or $\Phi$ with a small number of free parameters, then to use tracer samples to constrain the free parameters.

\section{ROTATION CURVES AND OVERALL MASS DISTRIBUTION}

The first piece of evidence is provided by the galactic rotation. There is a number of indications that in our galaxy, as well as in others of similar type, the bulk of the mass is roughly axisymmetric. Then, whatever tracer which follows the circular velocity provides one direct evidence for the radial force law through

$$
V_{c}^{2}=R \partial \Phi / \partial R \text {. }
$$

In the spherical approximation, the total mass within radius $R$ follows directly from

$$
M(R)=G^{-1} R V_{c}^{2}(R) .
$$

Even in this oversimplified approximation, what we get is just the total mass inside a sphere, with very little information about the distribution inside.

In order to determine what happens at distance $R$, one should face equation (5), which again requires the estimation of a local derivative, $\partial V / \partial R$ :

$$
\partial M / \partial R=(4 \pi G)^{-1} V^{2} / R^{3}(1+2(R / V) \partial V / \partial R) .
$$

Looking into the details of rotation velocity data (for instance in Rohlfs et al., 1986), it is clear that the large scale features of the rotation curve are reasonably well constrained, but existing data are far from tracing accurately velocity gradients at any radius. Therefore, most investigations in this field start with constructing mass models. An extensive review of this topic can be found in Binney and Tremaine (1988).

There are two separate questions involved in this dynamical modelling: one is the mathematical aspect, which we will adress briefly in the chapter dealing 
with Stäckel potentials, the other is the link between model parameter estimation and the constraints given by observations. Regarding the second question, recent models, such as the ones by Rohlfs and Kreitschmann (1988) or Haud and Einasto (1989), do improve the situation because they are based on improved knowledge of the rotation curve and improved rotation parameters, but there is no guarantee for the validity of the mathematical representation for other purposes than fitting the rotation curve. A striking illustration of this fact has been given by Bahcall, Soneira and Schmidt (1982), who show that a simplified analysis, based on single spherical component models, may overestimate the dynamical mass by a factor 2.6 within $10 \mathrm{kpc}$ and still 1.2 within $20 \mathrm{kpc}$, irrespective of the quality of the fit of the rotation curve.

The link between model parameters and observational constraints has been investigated in great detail by Caldwell and Ostriker (1981). These authors include as observational constraints not only the rotation curve and the local rotation gradient, as defined by the Oort's constants A and B, but also the surface density of our galaxy at $R_{\odot}$. They also introduce remote constraints, such as the velocity distribution of distant globular clusters which requires an extended halo of very low luminosity matter.

In Bienaymé et al. (1987), we include both the rotation curve constraints and others derived from the vertical distribution of disc stars in a single iterative solution. Details of this solution are discussed in chapter 3.

From this very raw and partial survey of the rotation constraints I conclude that

a) remote constraints require an extended corona.

b) the innermost parts of the rotation curve require a massive central bulge.

c) the mass between bulge and corona should be distributed such that it produces a flat rotation curve at about $200 \mathrm{~km} \mathrm{~s}^{-1}$, from $5 \mathrm{kpc}$ outwards.

d) more details about how the mass involved in producing this rotation curve splits into different components should be derived from studies of the vertical structure of the galaxy.

e) a drastic improvement of the determination of the Oort's constant would be very important.

\section{THE VERTICAL STRUCTURE}

Here, again, a quick look at formulae that are valid in a simplified situation does illustrate the main problems. As long as the mass distribution can be represented by a series of infinite parallel layers, the potential can be separated in radial and vertical components

$$
\Phi=\Phi_{R}+\Phi_{z}
$$

This is a good approximation at moderate distances from the galactic plane in the neighbourhood of the sun. Moderate means $z \ll R_{\odot}$, which we will consider as satisfied below $1 \mathrm{kpc}$. The collisionless Boltzmann equation (2) can then be written 
separately for the $z$ component of the phase space distribution (the index $i$ refers to the chosen tracer population)

$$
v_{z} \cdot \partial f_{i}\left(v_{z}, z\right) / \partial z+\partial \Phi_{z} / \partial z \cdot \partial f_{i}\left(v_{z}, z\right) / \partial v_{z}=0
$$

This means that observing the phase space distribution $f_{i}$ of a tracer population in the direction of the galactic poles will give sufficient information on the vertical force law.

In case isothermal tracers can be identified (iosthermal means in this case $\partial \sigma_{i v z} / \partial z=0$, where $\sigma_{i v z}$ is the rms velocity), then there is a straightforward solution of equation (7) for the vertical component of the force law, known as $K_{z}$

$$
K_{z}=\partial \Phi_{z} / \partial z=-\sigma_{i v z}^{2} / \rho_{i} \partial \rho_{i} / \partial z .
$$

The phase space distribution is specified by the velocity dispersion $\sigma_{i v z}$ and the trend of the volume density $\rho_{i}(z)$.

Assuming that we do have a good (presumably spectral) criterion to identify the members of such an isothermal population, observations of the nearby members will give the velocity dispersion and the luminosity function. A spectral survey together with photometric observations towards the polar directions will give the apparent magnitude distribution $a_{i}(m)$. The observational effort required to produce the necessary spectral survey is so heavy that the work of Upgren (1962) has been the basis of most investigations over more than twenty years. From $a_{i}(m)$ and the luminosity function, the inversion of an integral equation will lead to $\rho_{i}(z)$. Here, again, the resulting $K_{z}$ is just as reliable as the density derivative is. An important by-product of the $K_{z}$ estimate is the dynamical estimate of the local volume density $\rho_{o}$, which follows from the Poisson equation in its simplified form:

$$
\partial K_{z} / \partial z=4 \pi G \rho_{o} .
$$

Clearly, $\rho_{o}$, which depends on the local derivative of $K_{z}$, is even more sensitive to the scarcity of most density tracers near the galactic plane.

The different aspects of this quest have been reviewed in a colloquium held in Danbury one year ago (Davis Philip and Lu, 1989). A sample of the many $K_{z}$ curves obtained over nearly fifty years is printed on the cover of the colloquium proceedings. I do not like at all this picture, which conveys the impression that any new result in this field is definitely condemned to add to the general confusion. The reason for this confusion is that very little effort has been made to put meaningful error bars on the results. Most investigations based on the direct numerical estimation of the tracer density from star counts produce $K_{z}$ curves that steeply rise in the first $100 \mathrm{pc}$ and then abruptly fall beyond. A closer look at the tracer sample shows that the density law responsible for the steep rise is derived from one or two dozen stars, which means that there is indeed no information on the slope at low $z$. This is well illustrated by the negative slopes of the $K_{z}$ curves beyond $100 \mathrm{pc}$, which are physically impossible (they would imply layers of negative mass). 
A synthetic approach that imposes the density laws of the tracers to be realistic (Oort 1932, Oort 1960, Hill 1960) does avoid this difficulty, although it should not be forgotten that imposing realistic mathematical shapes does not create information where there is no data to constrain them. At larger distances, the difficulties are to safely identify complete samples of tracer population members, and also to measure radial velocities of faint stars. As a result, it is uncertain whether the density tracers at large $z$ belong indeed to the isothermal component they are supposed to belong to. In addition, halo stars sometimes are not recognized. Beyond 1.5 $\mathrm{kpc}$, the plane parallel approximation is dramatically wrong. Also, both synthetic and direct numerical inversions are sensitive to errors in the luminosity function (absolute magnitude calibration). Three recent papers have substantially improved the situation.

\subsection{Bahcall (1984)}

used a mass model including a double exponential disc, a de Vaucouleurs spheroid, and a corona. He tentatively introduced different hypothetical unseen mass discs to reconcile the dynamical mass required to fit the tracer distributions with the observed mass.

His analysis was based on a self-consistent solution of the combined PoissonBoltzmann system for isothermal components in the presence of a spheroid. The tracer samples used were the Upgren's F-dwarfs and K-giants towards the North galactic pole (Upgren 1962). Furthermore, Bahcall rediscussed both the luminosity functions of the tracer stars and also the validity of the isothermal decomposition.

The $K_{z}$ curve resulting from Bahcall's best fit solution is presented in Figure 1. A major consequence of this result is that it implies an unseen mass disc as heavy as the visible one: the total local mass density is found to be about 0.2 solar mass per cubic parsec, which is about twice what we actually see, and requires ten times more local dark matter density than the corona imposed by the rotation curve. Two investigations by Crézé et al. (1989) and by Gould (1989) lead to the same conclusions: the very large amount of missing mass in the solar neighbourhood found by Bahcall is a result of the poor constraints imposed by the scarce tracer data at low $z$. In other words, a model with no unseen mass disc would have fitted the raw data as well as the one chosen.

\subsection{Bienaymé, Robin, and Crézé (1987)}

followed a slightly different approach. Admitting that it is not easy to identify members of a predefined isothermal component of the galaxy, they avoided the difficulty by using a complete decomposition of all not-too-young disc stars in coeval isothermal components.

The basic mass model is not very different from Bahcall's, only the stellar disc is made of a series of Einasto ellipsoids, each associated with an age range. Then a scenario of galaxy evolution (including star formation, stellar evolution and progressive heating of the disc) is used to predict the absolute magnitude and colour 


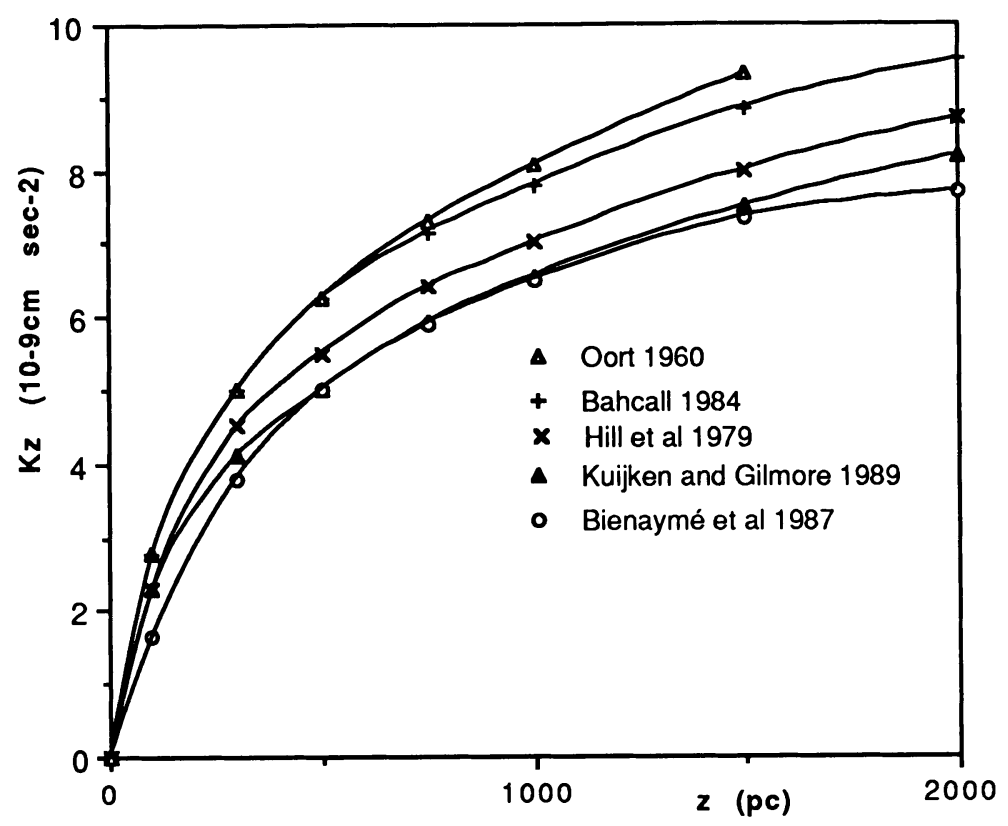

Figure 1. The galactic force law perpendicular to the galactic plane.

distribution in each coeval population (Robin and Crézé 1986). Then the whole disk population can be split into a series of isothermal components, each component being associated to an age. The reasonably well known age/velocity dispersion relation in the solar neighbourhood (Mayor 1974) is used to assign each component an isothermal velocity distribution. The dynamical closure ties the characteristic scales of the density ellipsoids to the velocity dispersions through the potential.

This approach is based on

- the idea that stars born at the same epoch should have experienced the same dynamical history

- the observation that in the solar neighbourhood, whenever a good age criterion enables selection of a good coeval sample, the velocity distribution turns out to be gaussian.

The main consequence of this approach is that it makes general star counts a tracer of the potential, because in this global modelling we do not need to know which among the observed stars belong to a certain component. Only the overall magnitude distribution of the model predictions should fit the observed one. Also, the modelling being three-dimensional, samples at intermediate galactic latitudes can be used to set additional constraints. The samples are far larger than the usual tracer samples and the statistical errors are negligable. Systematic errors in the luminosity function for stars taken from the whole HR diagram or errors in 
assigning velocity dispersions to subpopulations, can hardly be suspected to affect the resulting $K_{z}$. No selection error can be made in the observation of the tracers (as long as the disc dominates).

The resulting $K_{z}$ is given in Figure 1. It requires much less mass than the $K_{z}$ distributions derived by Bahcall and by Oort. Near the galactic plane, the local volume density is $0.1 \pm 0.01$ solar mass per cubic parsec, which is in good agreement with the observed mass.

This result is not very well established beyond say $1500 \mathrm{pc}$, because the dynamical modelling is not fully three-dimensional, even though the mass model is. Furthermore, beyond this distance from the midplane the contribution of non-disc stars becomes important and the prediction of this contribution in the model is not very well constrained. The result has been questioned, mainly because it involves a lot of modelling.

\subsection{Kuijken and Gilmore (1989)}

produced a new survey of K-dwarfs towards the south galactic cap. New photometric and radial velocity data have been obtained for several hundreds of stars over an extended range in $z$. Their approach involves nearly no modelling hypothesis, although substantial progress has been made in the theoretical analysis. They adopted a simple parameterization of the potential (instead of modelling the densities). Then they directly derived the velocity distribution of the tracer stars from their space distribution in each hypothetical potential according to equation (7). Hence they avoided the problem of isothermal decomposition. The likelihood of the observed velocity distribution for a certain model is used to choose the best potential.

The resulting $K_{z}$ is presented in Figure 1. It is not significantly different from the distribution obtained by Bienaymé et al. The KG sample is again too scarce at low $z$ to provide a good constraint on the local volume density. Above $1500 \mathrm{pc}$, both approaches suffer from not being fully three-dimensional. The local surface densities obtained are quite similar and both results do not require any hidden mass in the solar neighbourhood.

Hence, two independent results, obtained by Bienaymé et al. (1987) and Kuijken and Gilmore (1989), based on different data and different theoretical approaches, are in such a good agreement that one can hardly escape the conclusion that substantial progress has been made in the knowledge of the vertical component of the galactic potential, at least below $1500 \mathrm{pc}$.

\section{STACKEL POTENTIALS}

The final step to fully understand the galactic potential requires full threedimensional modelling that is valid at any $z$. The solution may be found in the Stäckel potentials. One important property of these potentials is that they are separable in spheroidal coordinates, which means that one-dimensional solutions 
of the Boltzmann equation can be worked out without any loss of generality for spheroidal systems. They are already being extensively used in the study of triaxial ellipticals. Their axisymmetric oblate version, which would be suitable for discs, has been studied by Eddington (1915), Kuzmin (1953), Hori (1962), Van de Hulst (1962), Dejonghe and de Zeeuw (1988). They have been shown to fit (at least locally) any general potential with good precision, and to provide integrals of motion (de Zeeuw and Lynden Bell 1985). They are suitable to address the $K_{z}$ problem (Statler 1989), because they release the limitations imposed by the planeparallel approximation and they give a good formalism to analyse distributions in the phase space.

There are, however, two limitations which make the final solution still steps ahead. An important constraint for choosing the appropriate potential out of the extensive Stäckel family is still missing. We should know what the velocity ellipsoid looks like at high $z$. Another limitation comes from the unfriendly mathematics involved.

\section{REFERENCES}

Bahcall, J., Soneira R., Schmidt, M. (1982) Ap. J. 258, L23.

Bahcall, J. (1984a) Ap. J. 276, 156.

Bahcall, J. (1984b) Ap. J. 276, 169.

Bienaymé, O., Robin, A.C., Crézé, M. (1987) Astron. Astrophys. 180, 94.

Binney J., Tremaine, S. (1987) " Galactic Dynamics " , Princeton Univ. Press, Princeton.

Caldwell, J.A.R. , Ostriker, J.P. (1981) Ap. J. 251, 61.

Crézé, M. ,Bienaymé, O.,Robin, A.C. (1989) Astron. Astrophys. 211, 1.

Davis Philip, A.G., Lu, P., (Eds) (1989) "The gravitational force perpendicular to the galactic plane ", L.Davis Press Schenectady, New York.

Dejonghe, H., de Zeeuw, T. (1988) Ap. J. 329, 720

de Zeeuw, T.,, Lynden Bell, D. (1985) M.N.R.A.S. 215, 599

Eddington, A.S. (1915) M.N.R.A.S., 76, 37

Einasto, J., Haud U. (1989) Astron. Astrophys. 223, 89.

Gould A., (1989) in "The gravitational force perpendicular to the galactic plane", L.Davis Press Schenectady, New York, p 19

Haud U., Einasto, J. (1989) Astron. Astrophys. 223, 95.

Hill, G. Hilditch, R., Barnes, J.V. (1979) M.N.R.A.S. 186, 813.

Hori, G. (1962) P.A.S.J. 14, 353

Kuijken, K. and Gilmore, G. (1989) M.N.R.A.S. 239, 571

Kuijken, K. and Gilmore, G. (1989) M.N.R.A.S. 239, 605

Kuijken, K. and Gilmore, G. (1989) M.N.R.A.S. 239, 651

Kuzmin, G. (1953) Tartu Astr. Obs. Teated, 1,1

Mayor, M.,(1974) Astron. Astrophys. 32, 321.

Oort , J. (1932) Bull. Astron. Inst. Netherlands 6, 249.

Oort, J. (1960) Bull. Astron. Inst. Netherlands 15, 45.

Robin, A., Crézé M. (1986) Astron. Astrophys. 157, 71.

Robin, A.C., Crézé, M. ,Bienaymé, O. (1988) in XXIIIth Rencontre de Moriond, "Dark Matter", Eds. J. Audouze and J. Tran Thanh Van, p 239. 
Rohlfs, K., Chini, R., Wink, J.E., Böhme, R.(1986) Astron. Astrophys., 158, 181

Rohlfs, K., Kreitschmann, J. (1988) Astron. Astrophys., 201, 51

Statler , T. (1989) Ap. J. 344, nnn.

Upgren, A.R. (1962) Astron. J. 67, 37.

Upgren, A.R. (1978) Astron. J. 83, 626.

Van de Hulst, H.C. (1962) Bull. Astron. Inst. Netherlands 16, 235 


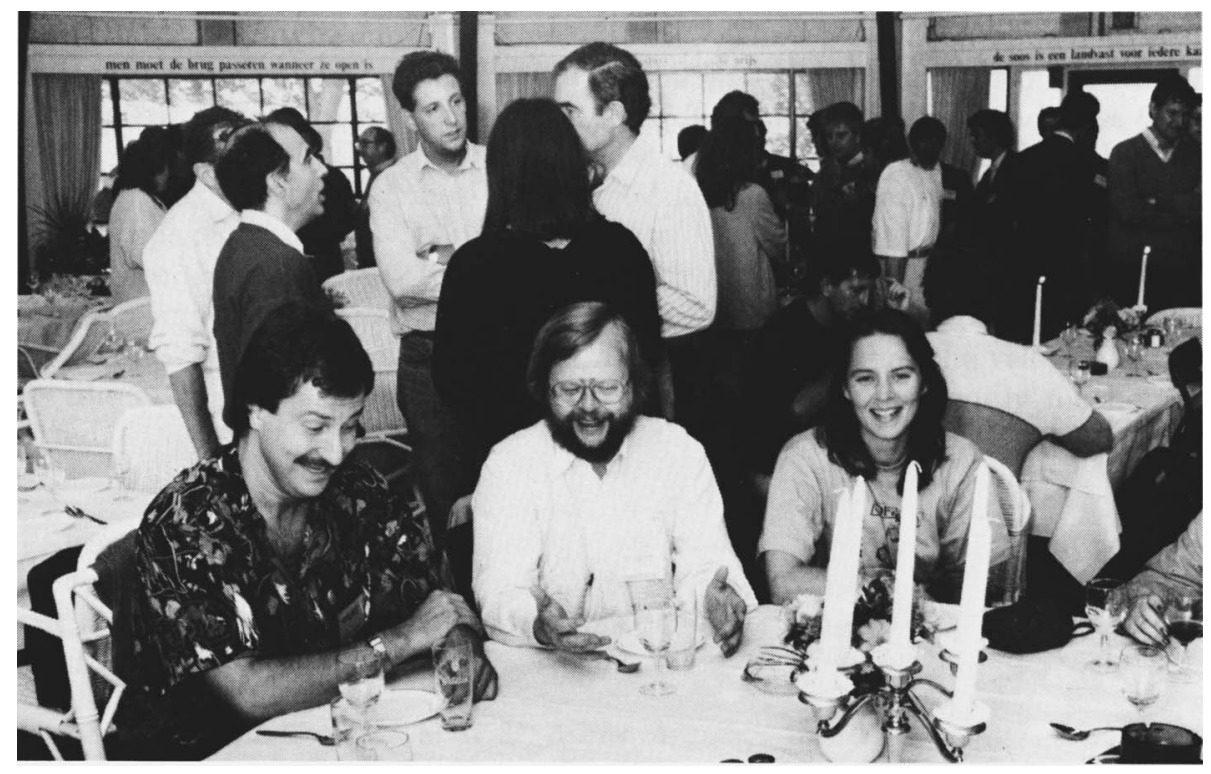

